University of Nebraska - Lincoln

DigitalCommons@University of Nebraska - Lincoln

Timothy J. Gay Publications

Research Papers in Physics and Astronomy

July 1987

\title{
Saddle-point electrons in ionizing ion-atom collisions
}

R. E. Olson

University of MissouriRolla, Rolla, Missouri

Timothy J. Gay

University of Nebraska - Lincoln, tgay1@unl.edu

H. G. Berry

Argonne National Laboratory, Argonne, Illinois

V. D. Irby

University of MissouriRolla, Rolla, Missouri

Follow this and additional works at: https://digitalcommons.unl.edu/physicsgay

Part of the Physics Commons

Olson, R. E.; Gay, Timothy J. ; Berry, H. G.; and Irby, V. D., "Saddle-point electrons in ionizing ion-atom collisions" (1987). Timothy J. Gay Publications. 26.

https://digitalcommons.unl.edu/physicsgay/26

This Article is brought to you for free and open access by the Research Papers in Physics and Astronomy at DigitalCommons@University of Nebraska - Lincoln. It has been accepted for inclusion in Timothy J. Gay Publications by an authorized administrator of DigitalCommons@University of Nebraska - Lincoln. 
Phys. Rev. Lett. 59, 36 - 39 (1987)

[Issue 1 - July 1987]

\title{
Saddle-point electrons in ionizing ion-atom collisions
}

\author{
R. E. Olson and T. J. Gay \\ Laboratory for Atomic \& Molecular Research and Physics Department, \\ University of MissouriRolla, Rolla, Missouri 65401 \\ H. G. Berry \\ Physics Division, Argonne National Laboratory, Argonne, Illinois 60439 \\ E. B. Hale and V. D. Irby \\ Physics Department, University of MissouriRolla, Rolla, Missouri 65401
}

\section{Received 30 January 1987}

We have studied $\mathrm{H}^{+}+\mathrm{He}$ ionizing collisions, and find that electrons stranded between the two postcollision Coulomb centers dominate the ejected-electron spectra for intermediate $(\sim 100 \mathrm{keV})$ projectile energies. The importance of these electrons is clearly established by our classical calculations which are in qualitative agreement, in both shape and magnitude, with experimental spectra taken by us, and with earlier measurements. These "saddle-point" electrons are produced by a classically understandable mechanism, and are unambiguously observed at nonzero scattering angles.

C) 1987 The American Physical Society

URL: http://link.aps.org/abstract/PRL/v59/p36

DOI: 10.1103/PhysRevLett.59.36

PACS: $34.50 . \mathrm{Fa}$ 


\title{
Saddle-Point Electrons in Ionizing Ion-Atom Collisions
}

\author{
R. E. Olson and T. J. Gay \\ Laboratory for Atomic and Molecular Research and Physics Department, \\ University of Missouri-Rolla, Rolla, Missouri 65401 \\ H. G. Berry \\ Physics Division, Argonne National Laboratory, Argonne, Illinois 60439 \\ and \\ E. B. Hale and V. D. Irby \\ Physics Department, University of Missouri-Rolla, Rolla, Missouri 65401 \\ (Received 30 January 1987)
}

\begin{abstract}
We have studied $\mathrm{H}^{+}+\mathrm{He}$ ionizing collisions, and find that electrons stranded between the two postcollision Coulomb centers dominate the ejected-electron spectra for intermediate $(\simeq 100 \mathrm{keV})$ projectile energies. The importance of these electrons is clearly established by our classical calculations, which are in qualitative agreement, in both shape and magnitude, with experimental spectra taken by us, and with earlier measurements. These "saddle-point" electrons are produced by a classically understandable mechanism, and are unambiguously observed at nonzero scattering angles.
\end{abstract}

PACS numbers: $34.50 . \mathrm{Fa}$

Electrons produced in ionizing collisions are generally associated either with the ionized target or with the receding projectile. In the first case, target electrons can be removed impulsively and placed into a continuum state ("impact" ionization), or into an autoionizing state of the target. In the latter case, the electron is captured into a continuum or autoionizing state of the projectile via "charge transfer to the continuum" (CTC) or "transfer ionization." Essentially all discussions of ionization in the literature have been based on this standard picture. In this Letter we present calculations and measurements to demonstrate that electrons stranded between the two nuclei make a dominant contribution to intermediate-energy ionizing collisions.

Several years ago in theoretical studies of the ionization of atomic hydrogen by protons at intermediate energies, Olson ${ }^{1}$ observed a significant enhancement of the number of electrons with post-collision velocities close to $\mathbf{v}_{p} / 2$, where $\mathbf{v}_{p}$ is the velocity of the projectile. He attributed this enhancement to the "stranding" of electrons on the transitory saddle region of the electric potential between the two protons. More recently, again in calculations of $\mathrm{H}^{+}+\mathrm{H}$ ionizing collisions, but at low energies $(E \leq 15 \mathrm{keV})$, Winter and $\mathrm{Lin}^{2}$ found that the inclusion of an additional basis set placed midway between the protons led to dramatic improvement in the agreement between their calculations and the measured total cross sections of Fite et al. ${ }^{3}$ Implicit in this improvement is the existence of a significant fraction of ionized electrons whose velocities are approximately half that of the projectile. In a paper last year, ${ }^{4}$ Olson gave details of the electron energy distributions for his earlier calculations.

Recently, Meckbach et al. ${ }^{5}$ discussed several points with respect to this problem and concluded, following the reanalysis of relative cross sections taken by Meckbach, Nemirovsky, and Garibotti, ${ }^{6}$ that electrons associated with the Coulomb saddle point would be seen in velocity space as a ridge stretching between the poles of electron population at $\mathbf{v}_{e}=0$ and $\mathbf{v}_{e}=\mathbf{v}_{p}$. Absent from their measurements on $\mathrm{He}$ ionization by protons,

$$
\mathrm{H}^{+}+\mathrm{He} \rightarrow \mathrm{H}^{+}+\mathrm{He}^{+}+e^{-}
$$

was information about ejected electrons in velocity space near $\mathbf{v}_{e}=\mathbf{v}_{p} / 2$, and at angles greater than $2.5^{\circ}$. In contrast, we will show that the saddle-point electrons are a global phenomenon which dominate ionization cross sections at intermediate energies. The saddle-point mechanism is not to be confused with "Wannier" ionization, valid only near threshold for electrons ejected at $0^{\circ} .{ }^{7}$ In this Letter, we report unambiguous observations of saddle-point electrons, and present absolute theoretical calculations which are in qualitative agreement, both in shape and magnitude, with our measurements as well as those of Rudd and collaborators. ${ }^{8-10}$

The saddle-point electrons are due to a classically understandable phenomenon, in which the electric field of the projectile pulls the electron out of the target atom, but moves on, leaving it stranded between the two postcollision Coulomb centers. Thus, to study reaction (1) we have employed the three-body classical-trajectory Monte Carlo (CTMC) method ${ }^{11}$ using the independentelectron model. ${ }^{12,13}$ An effective charge of 1.6875 and an ionization energy of 0.903 a.u. was used for the helium ion and atom, respectively. The CTMC method includes all the forces between the $\mathrm{H}^{+}, \mathrm{He}^{+}$, and $e^{-}$and inherently provides a consistent treatment of the ionization and charge transfer reactions. 
Figure 1 compares our calculated absolute ionized electron energy distribution, integrated over all angles, with the experimental results of Rudd and Madison ${ }^{10}$ for $100-\mathrm{keV}$ incident protons. We note that our calculated total ionization cross sections for reaction (1) are within $20 \%$ of the accepted values ${ }^{14}$ for energies between 60 and $500 \mathrm{keV}$ (at $100 \mathrm{keV}$ we are within $5 \%$ of the experimental values). Conspicuous in Fig. 1 is the lack of a maximum from the CTC component at $v_{e} \approx v_{p}$. It is apparent that although CTC is a major feature in the $0^{\circ}$ ejected electron spectra, it makes a minor contribution to the overall ionization process. We also note that the Born approximation reproduces the angle-integrated data quite well.

Since it is also clear from Fig. 1 that low-energy ejected electrons dominate the ionization process, as observed in other systems, ${ }^{15-17}$ a question naturally arises concerning the location of these electrons relative to the two nuclei. When the two nuclei were $50 a_{0}$ apart the postcollision space was divided into three regions of closest proximity to the projectile, the target nucleus, and the midpoint between the nuclei. We find most of the electrons in the central region to $200 \mathrm{keV}$ (see Table I); convergence of these results was confirmed at $100 a_{0}$. It is clear from Table I that these electrons contribute dominantly to the ionization cross section at intermediate energies. At similar energies the CTMC calculations ${ }^{1}$ for $\mathrm{H}^{+}+\mathrm{H}$ agree with the quantum-mechanical calculations of Shakeshaft ${ }^{18}$ for describing the CTC component of

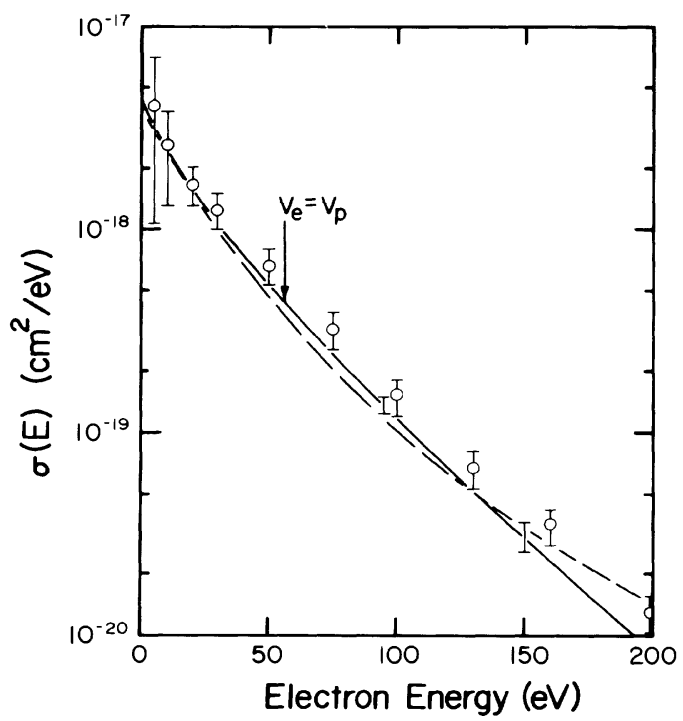

FIG. 1. Energy distribution of ejected electrons integrated over angle for $100-\mathrm{keV} \mathrm{H}^{+}+\mathrm{He}$ collisions. The solid line with two typical error bars is the result of our CTMC calculations. The dashed line is the Born-approximation calculation of Ref. 9. The data points are taken from Ref. 10. The energy (54.4 $\mathrm{eV})$ where the ejected-electron velocity matches the proton velocity is indicated. the ejected-electron distribution. Table I is also consistent with the $\mathrm{H}^{+}+\mathrm{H}$ calculations of Winter and Lin, ${ }^{2}$ which indicate the importance of saddle-point electrons at low collision energies.

To elucidate further the saddle-point ionization mechanism, we have made measurements of electron energy spectra at $17^{\circ}$ and $25^{\circ}$ for proton-helium ionizing collisions with incident proton energies of 60,100 , and 150 $\mathrm{keV}$. The apparatus has been described earlier. ${ }^{19}$ The proton beam was tightly collimated and crossed an effusive helium target in single-collision conditions. The electron energy analyzer is of the parallel-plate type, and can be used at angles of $0^{\circ}$, by virtue of an aperture in the analyzer back plate, or at angles between $17^{\circ}$ and $120^{\circ}$, a range dictated by analyzer and chamber geometry. The entrance aperture of the analyzer defines an acceptance angle of $\pm 4.5^{\circ}$. The magnetic field in the interaction volume was reduced to less than $10 \mathrm{mG}$ by Helmholtz coils and Mu-metal shields. Possible effects due to contact potentials, electron absorption in background gas, and a neutral beam fraction were studied and shown to be negligible. The relative detectoranalyzer efficiency versus electron energy was calibrated by normalizing to the $150-\mathrm{keV}$-energy spectrum at $30^{\circ}$ of Rudd and Jorgensen. ${ }^{8}$ Absolute error bars in our data are derived from the absolute errors discussed by Rudd and Madison ${ }^{10}$; relative errors in our energy spectra are less than $10 \%$.

In Fig. 2 we compare the CTMC calculations and our measurements at $100 \mathrm{keV}$ to the absolute cross sections of Rudd and Madison ${ }^{10}$ and interpolated data from Rudd and Jorgensen ${ }^{8}$ for electrons ejected at $13.6 \mathrm{eV}$ $\left(v_{e}=0.50 v_{p}\right), 30 \mathrm{eV}\left(v_{e}=0.74 v_{p}\right), 54.4 \mathrm{eV}\left(v_{e}=v_{p}\right)$, and $100 \mathrm{eV}\left(v_{e}=1.36 v_{p}\right)$. The classical calculations agree with the measurements to $35 \%$ for angles $\theta \leq 50^{\circ}$, with the differences increasing to a factor of 2 at larger angles. Plane-wave Born-approximation calculations 9,10 are available for $13.6,30$, and $100 \mathrm{eV}$ ejected-electron energies at $100 \mathrm{keV}$. Such a single-center calculation is clearly inadequate to describe the saddle-point electrons, as can be seen from the 13.6-eV $\left(v_{e}=0.50 v_{p}\right)$ and $30-\mathrm{eV}$ $\left(v_{e}=0.74 v_{p}\right)$ results in Fig. 2. However, the classical

TABLE I. Calculated ejected-electron flux after the collision assigned to the three regions of closest proximity to the target nucleus, midpoint between the nuclei, and the projectile nucleus.

\begin{tabular}{cccr}
\hline \hline & \multicolumn{3}{c}{ Flux fraction (\%) } \\
\hline$(\mathrm{keV})$ & Target & Midpoint & Projectile \\
\hline 60 & $19.1 \pm 0.9$ & $57.8 \pm 1.5$ & $23.1 \pm 1.0$ \\
100 & $24.5 \pm 0.8$ & $59.4 \pm 1.3$ & $16.1 \pm 0.7$ \\
200 & $46.8 \pm 1.4$ & $47.6 \pm 1.4$ & $5.6 \pm 0.5$ \\
300 & $61.1 \pm 1.6$ & $36.8 \pm 1.3$ & $2.1 \pm 0.3$ \\
500 & $74.8 \pm 2.0$ & $24.6 \pm 1.2$ & $0.6 \pm 0.2$ \\
\hline \hline
\end{tabular}




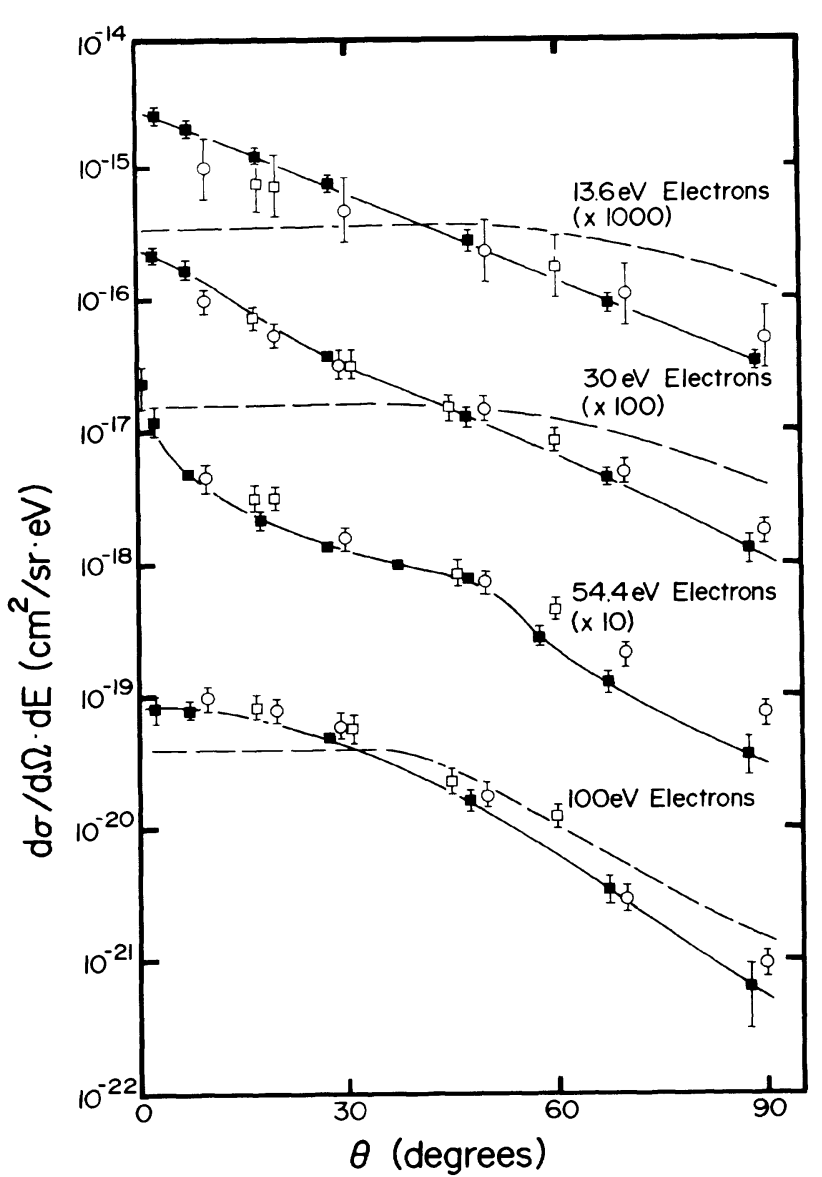

FIG. 2. Doubly differential cross sections for the angular distributions of electrons ejected in $100-\mathrm{keV} \mathrm{H}^{+}+\mathrm{He}$ collisions for $13.6,30,54.4$, and $100 \mathrm{eV}$. The filled squares are absolute values from our classical calculations. The solid lines serve to guide the eye. The open squares are our experimental data; the 13.6- and 54.4-eV points are normalized to interpolated values from Ref. 8 at $30^{\circ}$; the $30-$ and $100-\mathrm{eV}$ data are normalized to values from Ref. 10 at $20^{\circ}$. The open circles are absolute cross sections from Ref. 9 and interpolated values from Ref. 8. The dashed lines are the plane-wave Bornapproximation calculations (Refs. 9 and 10) for the 13.6-, 30-, and $100-\mathrm{eV}$ ejected electrons.

calculations are able to describe these data and also the CTC component of the scattering that manifests itself as a sharp rise at small angles for $54.5-\mathrm{eV}\left(v_{e}=v_{p}\right)$ ejected electrons. ${ }^{20}$

In order to observe clearly the dominant collision mechanisms, one must make observations at $\theta \neq 0^{\circ}$. By our doing so, the complications due to projectile-centered phenomena are removed. These phenomena are CTC and the possibility of autoionization in the projectile rest frame 21,22 is due to transfer ionization:

$$
\mathrm{H}^{+}+\mathrm{He} \rightarrow \mathrm{H}+\mathrm{He}^{++}+e^{-} \text {. }
$$

In Fig. 3 we display absolute doubly differential cross

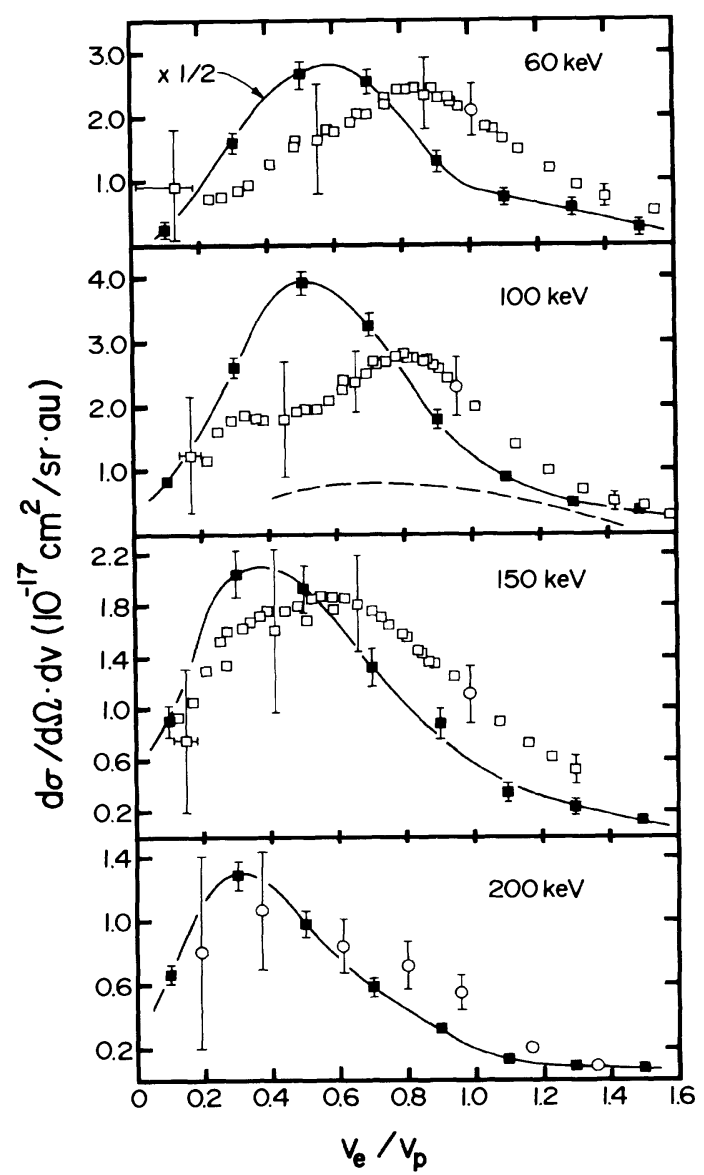

FIG. 3. Doubly differential cross sections in velocity space for electrons ejected at $17^{\circ}$ for $60-, 100-, 150-$, and $200-\mathrm{keV}$ protons. The filled squares are absolute values from our classical calculations. The open squares are our experimental data, normalized at $v_{e} \approx v_{p}$ to absolute values interpolated from the data of Ref. 8 (open circles). The open circles at $200 \mathrm{keV}$ are data of Ref. 9. The horizontal error bars represent uncertainty in the velocity due to the effects of contact potentials.

sections for electron scattering to $17^{\circ}$ plotted in velocity space for proton energies from 60 to $200 \mathrm{keV}$. Similar data at $25^{\circ}$ were observed. Such plots, which are representative of electron scattering at nonzero angles, clearly show the importance of the saddle-point electrons in the electron spectra. The calculations displayed in Fig. 3 are in qualitative agreement in shape and absolute magnitude with the characteristic saddle-point electron peak between $v_{e}=0$ and $v_{e}=v_{p}$, except at $60 \mathrm{keV}$ where the ratio of the proton-to-helium electron velocities are at the lower limit of the validity of the CTMC method. ${ }^{23}$ We note that the maximum of the electron spectra shown in Fig. 3 increases from $v_{e} / v_{p}$ of 0.4 to 0.8 as the proton energy is decreased from 200 to $60 \mathrm{keV}$. This is consistent with the results of Table I which demonstrate the increasing importance of projectile-centered electrons in the total ionization cross sections. 
We also report the calculated relative contribution to the total ionization cross section for scattering into a $\pm 1^{\circ}$ acceptance angle at $0^{\circ}$ and $17^{\circ}$; for a $100 \mathrm{keV}$ proton energy, we have integrated the cross sections over velocity and find a contribution of $2.6 \times 10^{-19} \mathrm{~cm}^{2}$ at $0^{\circ}$ and $3.6 \times 10^{-18} \mathrm{~cm}^{2}$ at $17^{\circ}$. Our calculated total ionization cross section is $9.6 \times 10^{-17} \mathrm{~cm}^{2}$. This calculation illustrates that a small cross section associated with projectile-centered phenomena can complicate an accurate theoretical analysis of $0^{\circ}$ electron ejection. As an example, the transfer ionization reaction is known ${ }^{21,22}$ to dominate the production of projectile-centered electrons for multiply charged ions colliding with $\mathrm{He}$ at $100 \mathrm{keV} / \mathrm{u}$. A minor component of these events for $\mathrm{H}^{+}+\mathrm{He}$ collisions will complicate the analysis of the $0^{\circ}$ cusp structure. For $\mathrm{H}^{+}+\mathrm{He}$ collisions, the transfer ionization cross section $^{24}$ is $8.0 \times 10^{-19} \mathrm{~cm}^{2}$ at $100 \mathrm{keV}$, and recent measurements by Gibson and Reid ${ }^{25}$ measure the CTC cross section to be $6.1 \times 10^{-19} \mathrm{~cm}^{2}$ at this energy. It should also be noted that our $0^{\circ}$ calculations and measurements are consistent with the data of Mekbach, Nemirovsky, and Garibotti ${ }^{6}$ and display the asymmetry in the peak associated with charge transfer to the continuum.

Thus, in conclusion, we have presented calculations and experimental data that clearly show the importance of saddle-point electrons in ionizing collisions. These electrons dominate the collisions to unexpectedly high energies, yielding characteristic maxima in the velocity spectra at nonzero angles. This scattering phenomenon is inherently classical and has been delineated with use of CTMC calculations.

The authors gratefully acknowledge the support by the U.S. Department of Energy [the Office of Fusion Energy (R.E.O. and V.D.I.) and the Office of Basic Energy Sciences (H.G.B.)] and by the Physics Division of the National Science Foundation (T.J.G.).
${ }^{1}$ R. E. Olson, Phys. Rev. A 27, 1871 (1983).

${ }^{2}$ T. G. Winter and C. D. Lin, Phys. Rev. A 29, 3071 (1984).

${ }^{3}$ W. L. Fite, R. F. Stebbings, D. G. Hummer, and R. T. Brackmann, Phys. Rev. 119, 663 (1960).

${ }^{4}$ R. E. Olson, Phys. Rev. A 33, 4397 (1986).

${ }^{5}$ W. Meckbach, P. J. Focke, A. R. Goni, S. Suarez, J. Macek, and M. G. Menendez, Phys. Rev. Lett. 57, 1587 (1986).

${ }^{6}$ W. Meckbach, I. B. Nemirovsky, and C. R. Garibotti, Phys. Rev. A 24, 1793 (1981).

${ }^{7}$ G. H. Wannier, Phys. Rev. 90, 817 (1953).

${ }^{8}$ M. E. Rudd and T. Jorgensen, Jr., Phys. Rev. 131, 666 (1963).

${ }^{9}$ M. E. Rudd, C. A. Sautter, and C. L. Bailey, Phys. Rev. 151, 20 (1966).

${ }^{10}$ M. E. Rudd and D. H. Madison, Phys. Rev. A 14, 128 (1976).

${ }^{11}$ R. E. Olson and A. Salop, Phys. Rev. A 16, 531 (1977).

12 J. H. McGuire and L. Weaver, Phys. Rev. A 16, 41 (1977).

${ }^{13}$ R. E. Olson, J. Phys. B 12, 1843 (1979).

${ }^{14}$ M. E. Rudd, Y. K. Kim, D. H. Madison, and J. W. Gallagher, Rev. Mod. Phys. 57, 965 (1985).

15 J. T. Park, J. E. Aldag, J. M. George, J. L. Peacher, and J. H. McGuire, Phys. Rev. A 15, 508 (1977).

${ }^{16}$ T. L. Criswell, L. H. Toburen, and M. E. Rudd, Phys. Rev. A 16, 508 (1977).

${ }^{17}$ M. E. Rudd, Phys. Rev. A 20, 787 (1978).

${ }^{18}$ R. Shakeshaft, Phys. Rev. A 18, 1930 (1978).

${ }^{19}$ P. W. Arcuni, Ph.D. thesis, University of Chicago, 1985 (unpublished), and Phys. Rev. A 33, 105 (1986).

${ }^{20}$ I. A. Sellin, in Physics of Electronic and Atomic Collisions, edited by S. Datz (North-Holland, Amsterdam, 1982), pp. 195-221.

${ }^{21}$ L. H. Andersen, M. Frost, P. Huelplund, H. Knudsen, and S. Datz, Phys. Rev. Lett. 52, 518 (1984).

${ }^{22}$ S. Datz, C. Bottcher, L. H. Andersen, P. Hvelplund, and H. Knudsen, Nucl. Instrum. Methods B10/11, 116 (1985).

${ }^{23}$ R. E. Olson and A. Salop, Phys. Rev. A 16, 531 (1977).

${ }^{24}$ M. B. Shah and H. B. Gilbody, J. Phys. B 18, 899 (1985).

${ }^{25}$ D. K. Gibson and I. D. Reid, J. Phys. B 19, 3265 (1986). 